Supporting Information

\title{
Metabolic activation of pirfenidone mediated by cytochrome P450s and sulfotransferases
}

Shenzhi Zhou, ${ }^{\dagger}$ Wei Li,${ }^{\dagger}$ Min Tian, ${ }^{\dagger}$ Na Zhang,,${ }^{\dagger}$ Xiaojing Yang, ${ }^{\dagger}$ Weiwei Li,,$*$ Ying Peng, ${ }^{* \dagger}$ and Jiang Zheng* ${ }^{* \dagger \$}$

†Wuya College of Innovation, Shenyang Pharmaceutical University, Shenyang, Liaoning 110016, P. R. China

*State Key Laboratory of Functions and Applications of Medicinal Plants, Guizhou

Provincial Key Laboratory of Pharmaceutics, Guizhou Medical University, Guiyang, Guizhou 550025, P. R. China

$\S$ Key Laboratory of Environmental Pollution, Monitoring and Disease Control (J.Z.), Ministry of Education, Guizhou Medical University, Guiyang, 550025, P. R. China

Contents:

NAC conjugate 11 generated via sulfation (Figure S1); biliary NAC conjugate 11 (Figure S2); P450 enzymes responsible for formation of 2 (Figure S3); toxicity data (Figures S4/S5/S6). 
Figure S1
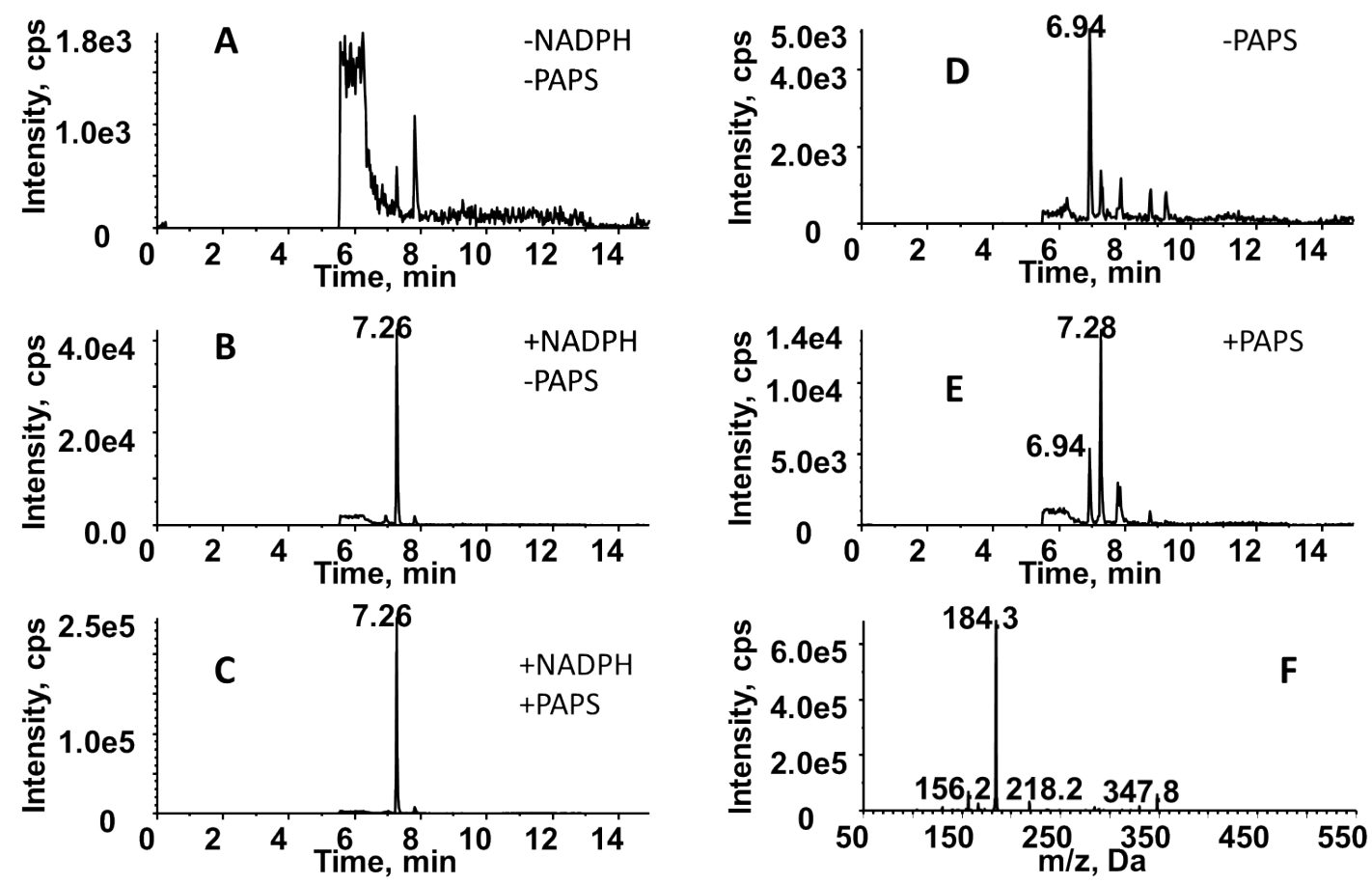

Figure S1. Characterization of NAC conjugate 11 generated in RLC and RLM-RLC incubation systems. Extracted ion $(m / z$ 347/184) chromatograms obtained from LCMS/MS analysis of NAC-fortified RLM-RLC incubations of 1 without NADPH or PAPS (A), with NADPH but without PAPS (B), and with NADPH and PAPS (C). Extracted ion ( $\mathrm{m} / \mathrm{z}$ 491/184) chromatograms obtained from LC-MS/MS analysis of RLC incubations containing 2 and NAC in the absence (D) or presence (E) of PAPS. F: MS/MS spectrum of NAC conjugate $\mathbf{1 1}$ produced in 2-fortified RLC incubations. 
Figure S2
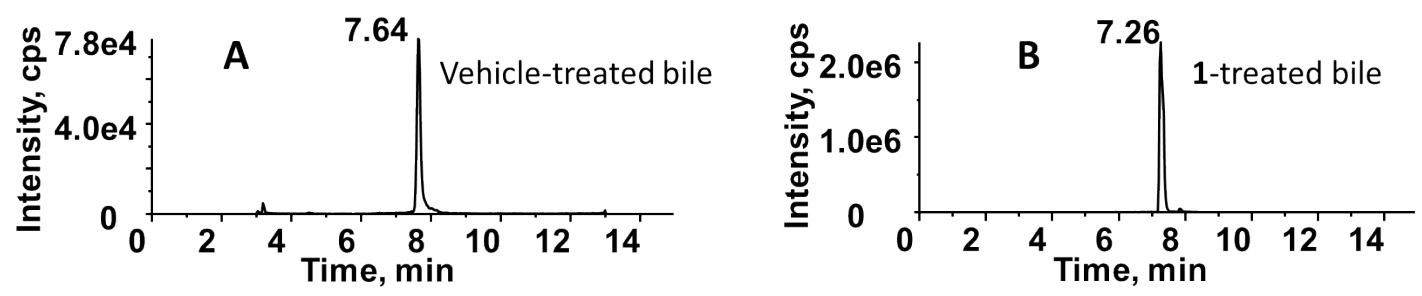

Figure S2. Characterization of biliary NAC conjugate 11. Extracted ion $(\mathrm{m} / \mathrm{z} 347 / 184)$ chromatograms obtained from LC-MS/MS analysis of bile from vehicle-treated (A) and 1-treated (B) rats. 


\section{Figure S3}
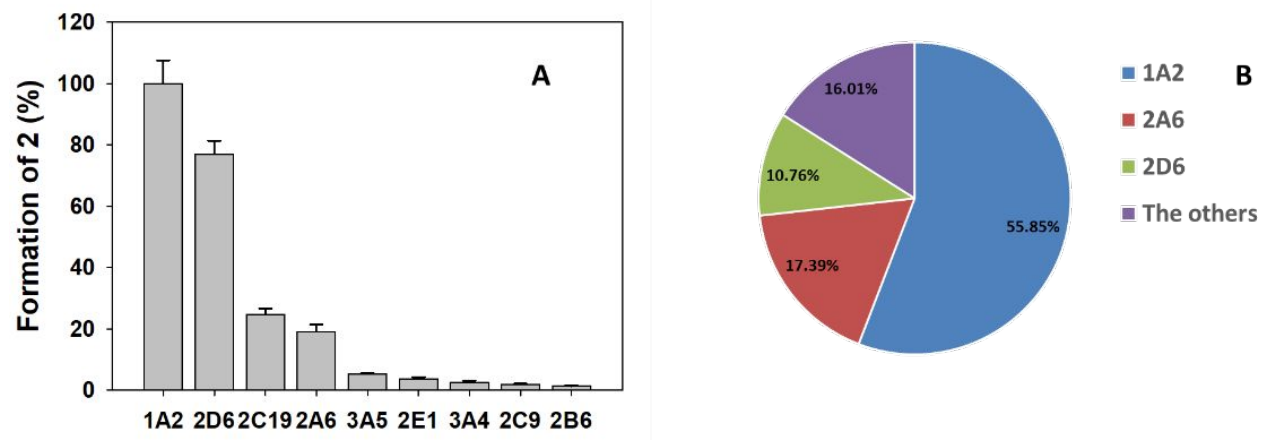

Figure S3. Role of individual human recombinant P450 enzymes in formation of oxidative metabolite $\mathbf{2}$. A: Formation of $\mathbf{2}$ in incubation of $\mathbf{1}$ with nine individual human recombinant $\mathrm{P} 450$ enzymes was measured by LC-MS/MS. The most abundant metabolite 2 detected was normalized to $100 \%$; B: The contribution of each P450 enzyme was calculated based on the formation of $\mathbf{2}$ after normalization, according to the natural abundance of the corresponding P450 enzymes in human liver microsomes. Data represent the mean $\pm \mathrm{SD}(\mathrm{n}=3)$. 
Figure S4
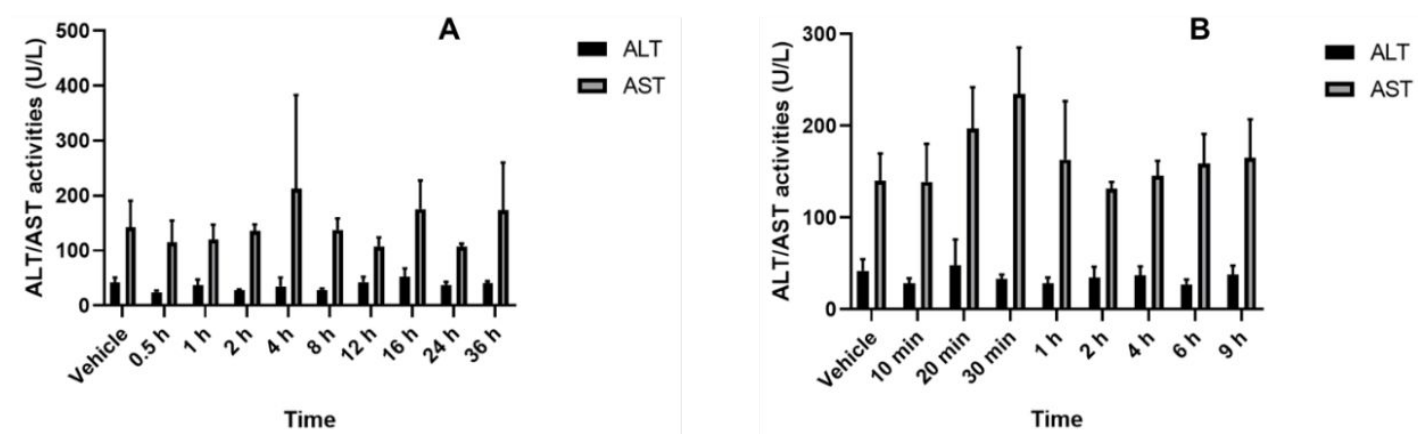

Figure S4. Time-course changes in serum ALT/AST activities of 1-treated mice. Mice were treated with $1(150 \mathrm{mg} / \mathrm{kg})$ or saline. The serum ALT/AST activities were measured at various time points. No significant hepatotoxicity was observed in 2 separate experiments (A and B). Data represent the mean $\pm \mathrm{SD}(\mathrm{n}=3)$. 


\section{Figure S5}

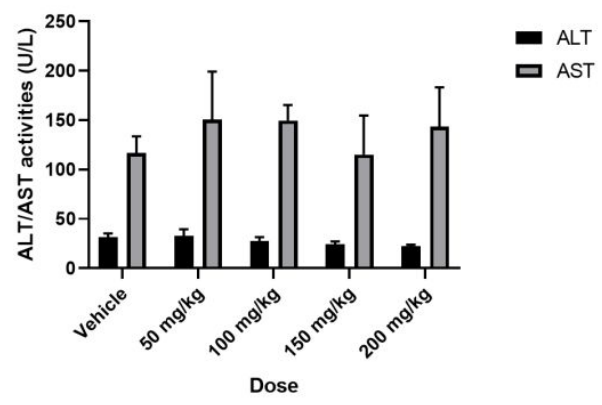

Figure S5. Serum ALT/AST levels of mice given various doses of 1 . Mice were treated with saline or 1 at various doses. The serum ALT/AST activities were measured after $0.5 \mathrm{~h}$ administration. Data represent the mean $\pm \mathrm{SD}(\mathrm{n}=3)$. 


\section{Figure S6}

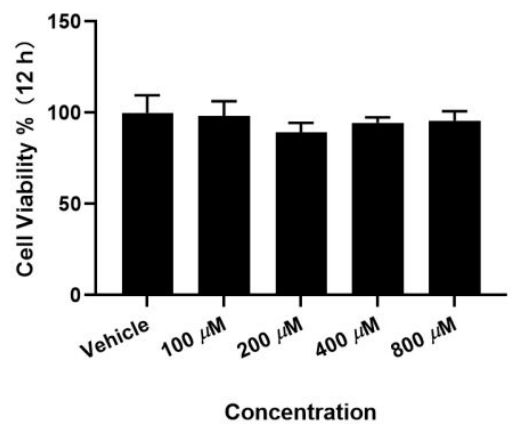

Figure S6. Cell viability of cultured primary hepatocytes treated with $\mathbf{1}$. Primary mouse hepatocytes were exposed to 1 at various concentrations. Cell viability was examined by MTT assay after $12 \mathrm{~h}$ exposure. Data represent the mean $\pm \mathrm{SD}(\mathrm{n}=3)$. 